\title{
E-LOCAL PSEUDOVARIETIES
}

\author{
A. MOURA
}

\begin{abstract}
Generalizing a property of the pseudovariety of all aperiodic semigroups observed by Tilson, we call E-local a pseudovariety $\mathrm{V}$ which satisfies the following property: for a finite semigroup, the subsemigroup generated by its idempotents belongs to $\mathrm{V}$ if and only if so do the subsemigroups generated by the idempotents in each of its regular $\mathcal{D}$-classes. In this paper, we present several sufficient or necessary conditions for a pseudovariety to be E-local or for a pseudoidentity to define an E-local pseudovariety. We also determine several examples of the smallest E-local pseudovariety containing a given pseudovariety.
\end{abstract}

\section{INTRODUCTION}

The motivation for this work came from an exercise suggested by Pin [6] about a result from Tilson [10]. With the aim of finding a method for computing the complexity of a finite semigroup in terms of the structure of its subsemigroups, Tilson started by establishing a useful method for computing the group-complexity of a finite semigroup with at most two non-zero $\mathcal{D}$-classes. This led him to prove the following result: given a finite semigroup $S$, the subsemigroup $\langle E(S)\rangle$ is aperiodic if and only if, for every regular $\mathcal{D}$-class $D$ of $S$, the subsemigroup $\langle E(D)\rangle$ is aperiodic.

As a consequence of the work of Fitz-Gerald [4], we have that a regular semigroup is orthodox if and only if the product of idempotents of every regular $\mathcal{D}$-class of $S$ is idempotent. Thus, it suffices to analyze the property of the product of idempotents to be an idempotent on every regular $\mathcal{D}$-class to conclude the property for an arbitrary product of idempotents.

Much work has been done on the structure of idempotent-generated semigroups. So, it becomes interesting to determine the pseudovarieties $\mathrm{V}$ satisfying the following property: given $S \in \mathrm{S},\langle E(S)\rangle \in \mathrm{V}$ if and only if $\langle E(D)\rangle \in \mathrm{V}$, for each regular $\mathcal{D}$ class $D$ of $S$. We call $E$-local a pseudovariety with this property.

This paper is a contribution towards the complete characterization of E-local pseudovarieties. We start by recalling, in Section 2, some basics of the theory of pseudovarieties of semigroups, in particular, some results concerning the block operator B_ and the idempotent-generated subsemigroup of a semigroup. Section 3 concerns the study of E-local pseudovarieties: we observe some properties and examples, we present several sufficient conditions for a pseudovariety to be E-local and we show that, in certain cases, these conditions are also necessary, and we introduce a new operator, ${ }_{-}^{\mathrm{E}}$, where $\mathrm{V}^{\mathrm{E}}$ is the smallest $\mathrm{E}$-local pseudovariety containing a pseudovariety V. Finally, in Section 4, we present some more necessary or sufficient conditions for a pseudoidentity to define an E-local pseudovariety.

2000 Mathematics Subject Classification. 20M07.

Key words and phrases. Finite semigroup; pseudovariety; idempotent-generated; regular Dclass; operator; E-local. 


\section{Preliminaries}

We briefly recall some basics of the theory of pseudovarieties of semigroups. We recommend $[1,6,9]$ for a better understanding of this area.

Let $S$ be a semigroup. We denote by $E(S)$ the set of idempotents of $S$ and by $\langle E(S)\rangle$ the subsemigroup of $S$ generated by $E(S)$. More generally, $\langle X\rangle$ denotes the subsemigroup of $S$ generated by $X \subseteq S$. In case $S$ is finite, $s^{\omega}$ denotes the unique idempotent in the subsemigroup generated by a given $s \in S$.

Let $S$ be a finite semigroup and let $D$ be a regular $\mathcal{D}$-class of $S$. Consider the equivalence relation $\sim$ on the set of group elements of a regular $\mathcal{D}$-class $D$ of $S$ defined in the following way: given two group elements $a$ and $b$ of $D, a \sim b$ if and only if there exists an idempotent-chain $e_{0}, e_{1}, \ldots, e_{n-1}, e_{n}$ such that $a \mathcal{H} e_{0}$, $b \mathcal{H} e_{n}$, and either $e_{i} \mathcal{R} e_{i-1}$ or $e_{i} \mathcal{L} e_{i-1}$, for all $i \in\{1, \ldots, n\}$. A block of $D$ is the Rees quotient of the subsemigroup of $S$ generated by a $\sim$-class modulo the ideal consisting of the elements that are not in $D$. The blocks of $S$ are the blocks of its regular $\mathcal{D}$-classes. This notion have been first introduced in Graham [5] and we also refer the reader to [7] for the notion of block operator on pseudovarieties, that we will define in the following paragraphs.

A class of finite semigroups that is closed under taking subsemigroups, homomorphic images and finite direct products is called a pseudovariety and generally denoted by V. For example, $\mathrm{S}$ denotes the pseudovariety of all finite semigroups.

We may construct new pseudovarieties from known ones by applying operators to pseudovarieties. In this paper, we use the following operators on pseudovarieties:

- EV consists of all $S \in \mathrm{S}$ such that $\langle E(S)\rangle \in \mathrm{V}$;

- DV consists of all $S \in \mathrm{S}$ such that, for every regular $\mathcal{D}$-class $D$ of $S, D \in \mathrm{V}$;

- for a pseudovariety $\mathrm{H}$ of groups, $\overline{\mathrm{H}}$ consists of all $S \in \mathrm{S}$ such that every subgroup $G$ of $S$ belongs to H;

- BV consists of all $S \in \mathrm{S}$ such that, for every block $B$ of $S, B \in \mathrm{V}$;

- $V_{E}$ is the pseudovariety generated by the idempotent-generated semigroups of $\mathrm{V}$.

The last operator was introduced in Almeida and Moura [2] and we refer the reader to that paper as needed, but we opt to present here an easy lemma that will be used frequently in this paper:

Lemma 2.1 (Almeida and Moura [2]). The operator $-\mathrm{E}$ has the following properties, where $\mathrm{V}$ and $\mathrm{W}$ are arbitrary pseudovarieties:

(1) $\mathrm{V} \subseteq \mathrm{W}$ implies $\mathrm{V}_{\mathrm{E}} \subseteq \mathrm{W}_{\mathrm{E}}$;

(2) $(\mathrm{V} \cap \mathrm{W})_{\mathrm{E}} \subseteq \mathrm{V}_{\mathrm{E}} \cap \mathrm{W}_{\mathrm{E}}$;

(3) $\left(V_{E}\right)_{E}=V_{E}$;

(4) $(\mathrm{EV})_{\mathrm{E}}=\mathrm{V}_{\mathrm{E}}$;

(5) $\mathrm{E}\left(\mathrm{V}_{\mathrm{E}}\right)=\mathrm{EV}$.

The main aim of our study is the characterization of the E-local pseudovarieties. For this purpose, we need some results concerning idempotent-generated subsemigroups and blocks of such subsemigroups.

Lemma 2.2. For every pseudovariety $\mathrm{V}, \mathrm{BBV}=\mathrm{BV}$.

Lemma 2.3. Let $S \in \mathrm{S}$ and $X \subseteq E(S)$. Then $\langle E\langle X\rangle\rangle=\langle X\rangle$.

To prove that the idempotent-generated subsemigroup of a regular semigroup is also regular, Fitz-Gerald [4] uses a technique that consists in writing a product of 
idempotents of $\langle E(S)\rangle$ as a product of idempotents of $\langle E(D)\rangle$, for a regular $\mathcal{D}$-class $D$ of $S$. As a consequence, we have the following lemma whose statement and proof may be found in [9, Lemma 4.13.1], for example. It enables us to easily conclude the statement presented in the introduction that a regular semigroup $S$ is orthodox if and only if the product of idempotents of every regular $\mathcal{D}$-class of $S$ is idempotent.

Lemma 2.4. Let $S$ be a semigroup and let $s \in\langle E(S)\rangle$ be an element of a regular $\mathcal{J}$-class $J$ of $S$. Then, there exists an idempotent-chain $e_{1}, e_{2}, \ldots, e_{m} \in E(J)$ such that $s=e_{1} e_{2} \cdots e_{m}$. Hence $\langle E(S)\rangle \cap J=\langle E(J)\rangle \cap J$.

Corollary 2.5. Every finite semigroup $S$ has the following properties:

(1) Let a be a regular element of $\langle E(S)\rangle$. Then a is in a block of $D_{a}$, where $D_{a}$ is the regular $\mathcal{D}$-class of $S$ containing a.

(2) Let $B$ be a block of $\langle E(S)\rangle$. Then $B$ is also a block of $\langle E(D)\rangle$, for some regular $\mathcal{D}$-class $D$ of $S$.

(3) Given $X \subseteq E(S)$, the regular $\mathcal{D}$-classes of $\langle X\rangle$ have only one block.

Proof. (1) and (2) follow immediately from Lemma 2.4 and from the definition of block of $S$. Now, by Lemma 2.3 and by (1), we have that every regular element of $\langle E\langle X\rangle\rangle=\langle X\rangle$ is in a block of $\langle E\langle X\rangle\rangle=\langle X\rangle$ and we have (3).

Lemma 2.4 also enables us to prove that the product of the operators $E_{-}$and $B_{\text {_ }}$ is an idempotent operator, as we see in the following corollary.

Corollary 2.6. For every pseudovariety $\mathrm{V}$, EBEBV $=\mathrm{EBV}$.

Proof. Let $S \in$ EBEBV, i.e., for every block $B^{\prime}$ of $\langle E(B)\rangle$, with $B$ a block of $\langle E(S)\rangle$, $B^{\prime} \in \mathrm{V}$. By Lemma 2.4, $B=\langle E(B)\rangle$. Moreover, the blocks of $\langle E(B)\rangle=B$ are $B$ and the trivial semigroup, the last one if $B$ is not a $\mathcal{D}$-class. Thus, for every block $B$ of $\langle E(S)\rangle, B \in \mathrm{V}$, i.e., $S \in \mathrm{EBV}$. The converse follows from E_ and B_ being increasing operators.

A pseudoidentity is a formal equality $u=v$, where $u, v \in \bar{\Omega}_{A} \mathrm{~S}$, the set of $A$-ary implicit operations. We say that $S \in \mathrm{V}$ satisfies $u=v$, and we write $S \models u=v$, if $u_{S}=v_{S}$. Recall that an $A$-ary operation $u_{S}: S^{A} \rightarrow S$ has the following property: for every homomorphism $\varphi: S \rightarrow T$, with $S, T \in \mathrm{V}$, the following diagram commutes:

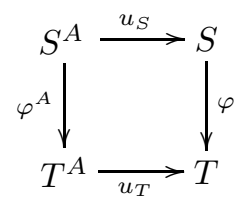

Reiterman's Theorem [8] says that every pseudovariety is defined by some set of finitary pseudoidentities, in the sense that it is the class of finite semigroups satisfying this set of pseudoidentities. The converse of the theorem is easily verified.

In this paper, we use, in particular, the pseudovarieties that we list below together with some corresponding bases of pseudoidentities defining them:

$$
\begin{aligned}
& \mathrm{I}=\llbracket x=y \rrbracket \quad \text { trivial semigroups; } \\
& \mathrm{J}=\llbracket(x y)^{\omega} x=(x y)^{\omega}=y(x y)^{\omega} \rrbracket \mathcal{J} \text {-trivial semigroups; } \\
& \mathrm{R}=\llbracket(x y)^{\omega} x=(x y)^{\omega} \rrbracket \quad \mathcal{R} \text {-trivial semigroups; }
\end{aligned}
$$




$$
\begin{aligned}
\mathrm{L} & =\llbracket y(x y)^{\omega}=(x y)^{\omega} \rrbracket & & \mathcal{L} \text {-trivial semigroups; } \\
\mathrm{A} & =\llbracket x^{\omega+1}=x^{\omega} \rrbracket & & \text { aperiodic (or } \mathcal{H} \text {-trivial) semigroups; } \\
\mathrm{G} & =\llbracket x^{\omega}=1 \rrbracket & & \text { groups; } \\
\mathrm{LG} & =\llbracket\left(x^{\omega} y\right)^{\omega} x^{\omega}=x^{\omega} \rrbracket & & \text { local groups; } \\
\mathrm{CR} & =\llbracket x^{\omega+1}=x \rrbracket & & \text { completely regular semigroups; } \\
\mathrm{CS} & =\llbracket x^{\omega+1}=x,(x y x)^{\omega}=x^{\omega} \rrbracket & & \text { completely simple semigroups; } \\
\mathrm{RB} & =\llbracket x^{2}=x, x y x=x \rrbracket & & \text { rectangular bands; } \\
\mathrm{LZ} & =\llbracket x y=x \rrbracket & & \text { left-zero semigroups; } \\
\mathrm{DA} & =\llbracket\left((x y)^{\omega} x\right)^{2}=(x y)^{\omega} x \rrbracket & & \text { regular } \mathcal{D} \text {-classes are aperiodic semigroups; } \\
\mathrm{DG} & =\llbracket(x y)^{\omega}=(y x)^{\omega} \rrbracket & & \text { regular } \mathcal{D} \text {-classes are groups; } \\
\mathrm{DO} & =\llbracket(x y)^{\omega}(y x)^{\omega}(x y)^{\omega}=(x y)^{\omega} \rrbracket & & \text { regular } \mathcal{D} \text {-classes are orthodox semigroups; } \\
\mathrm{DS} & =\llbracket\left((x y)^{\omega} x\right)^{\omega+1}=(x y)^{\omega} x \rrbracket & & \text { regular } \mathcal{D} \text {-classes are semigroups. }
\end{aligned}
$$

\section{E-LOCAL PSEUdOVARIETIES}

We start this section by observing some properties of E-local pseudovarieties and several examples of pseudovarieties having this property. In particular, we prove, in Example 3.8, the result of Tilson [10] that the pseudovariety A is E-local. After that, we present several sufficient conditions for a pseudovariety to be E-local and we show that these conditions are also necessary in case the pseudovariety is contained in EDS. We finish with the introduction of the operator ${ }_{-}^{\mathrm{E}}$, where $\mathrm{V}^{\mathrm{E}}$ denotes the smallest E-local pseudovariety containing $\mathrm{V}$.

3.1. Properties and examples. We start by noting that the property of being E-local is preserved under intersection. Next, we relate the E-locality of $\mathrm{V}, \mathrm{EV}$ and $\mathrm{V}_{\mathrm{E}}$.

Lemma 3.1. Let $\mathrm{V}$ be a pseudovariety and let $S \in \mathrm{S}$. The following conditions are equivalent:

(1) for every regular $\mathcal{D}$-class $D$ in $S,\langle E(D)\rangle \in \mathrm{V}$;

(2) for every regular $\mathcal{D}$-class $D$ in $S,\langle E(D)\rangle \in \mathrm{EV}$;

(3) for every regular $\mathcal{D}$-class $D$ in $S,\langle E(D)\rangle \in \mathrm{V}_{\mathrm{E}}$.

Proof. $(3) \Rightarrow(1) \Rightarrow(2)$ : This follows immediately from $\mathrm{V}_{\mathrm{E}} \subseteq \mathrm{V} \subseteq \mathrm{EV}$

$(2) \Rightarrow(3)$ : Note that $\langle E(D)\rangle \in \mathrm{EV}$ if and only if $\langle E\langle E(D)\rangle\rangle=\langle E(D)\rangle \in \mathrm{V}$, by Lemma 2.3. By the same lemma and by the definition of $\mathrm{V}_{\mathrm{E}}$, we deduce that $\langle E\langle E(D)\rangle\rangle=\langle E(D)\rangle \in \mathrm{V}_{\mathrm{E}}$.

Similarly, we may prove the following lemma:

Lemma 3.2. The following conditions are equivalent for every pseudovariety $\mathrm{V}$ and every finite semigroup $S$ :

(1) $\langle E(S)\rangle \in \mathrm{V}$;

(2) $\langle E(S)\rangle \in \mathrm{EV}$;

(3) $\langle E(S)\rangle \in \mathrm{V}_{\mathrm{E}}$.

The equivalence of E-locality for the pseudovarieties $V$, EV, and $V_{E}$ follows directly from the previous lemmas. 
Corollary 3.3. Let $\mathrm{V}$ be a pseudovariety. The following conditions are equivalent:

(1) $\mathrm{V}$ is E-local;

(2) $\mathrm{EV}$ is $\mathrm{E}$-local;

(3) $\mathrm{V}_{\mathrm{E}}$ is E-local.

The properties of the operator $-E$ (see Lemma 2.1) together with the previous corollary enable us to identify intervals consisting of E-local pseudovarieties.

Proposition 3.4. Let $\mathrm{V}$ be an $\mathrm{E}$-local pseudovariety. Then any pseudovariety $\mathrm{U} \in\left[\mathrm{V}_{\mathrm{E}}, \mathrm{EV}\right]$ is E-local.

Proof. Applying Lemma 2.1, we obtain $\mathrm{EV}=\mathrm{E}\left(\mathrm{V}_{\mathrm{E}}\right) \subseteq \mathrm{EU} \subseteq \mathrm{EV}$. The result now follows from Corollary 3.3.

In an attempt to identify all E-local pseudovarieties, we start by determining several families of pseudovarieties satisfying this property.

Proposition 3.5. Let $\mathrm{V}$ and $\mathrm{H}$, with $\mathrm{H} \subseteq \mathrm{G}$, be pseudovarieties. Then:

(1) BV is E-local;

(2) DV is E-local;

(3) $\overline{\mathrm{H}}$ is E-local.

Proof. (1) follows directly from item (2) of Corollary 2.5.

(2) By items (2) and (3) from Corollary 2.5, we have that, for every regular $\mathcal{D}$-class $D$ of $\langle E(S)\rangle$, there exists a regular $\mathcal{D}$-class $D^{\prime}$ of $S$ such that $D$ is a $\mathcal{D}$ class of $\left\langle E\left(D^{\prime}\right)\right\rangle$. Let $S$ be a semigroup such that, for every regular $\mathcal{D}$-class $D$, $\langle E(D)\rangle \in \mathrm{DV}$. Then, every regular $\mathcal{D}$-class of $\langle E(D)\rangle$ is a semigroup in $\mathrm{V}$. It follows that every regular $\mathcal{D}$-class of $\langle E(S)\rangle$ is a semigroup in $\mathrm{V}$.

(3) Let $S \in \mathrm{S}$ be such that, for every regular $\mathcal{D}$-class $D,\langle E(D)\rangle \in \overline{\mathrm{H}}$. Let $T$ be a subgroup of $\langle E(S)\rangle$. By Lemma 2.4, $T \subseteq\left\langle E\left(D_{T}\right)\right\rangle$, where $D_{T}$ is the $\mathcal{D}$-class of $S$ containing $T$. Hence $T \in \mathrm{H}$ and $\langle E(S)\rangle \in \overline{\mathrm{H}}$.

Example 3.6. Since J = DI (see Pin [6, Proposition III.4.1]), it follows from Proposition 3.5 that $\mathrm{J}$ is E-local.

Example 3.7. Since R = DLZ (see Pin [6, Proposition III.4.1]), it follows from Proposition 3.5 that $\mathrm{R}$ is E-local.

Example 3.8. To conclude the result from Tilson [10], it suffices to note that $A=\bar{I}$. So, the conclusion that the pseudovariety is E-local follows immediately from Proposition 3.5.

3.2. Characterizations. The properties of the operators $E_{-}$and $B_{-}$are useful to obtain the following sufficient and equivalent conditions for a pseudovariety to be E-local.

Proposition 3.9. The following conditions are equivalent:

(1) $\mathrm{EV}=\mathrm{EBEV}$;

(2) $\mathrm{EV}=\mathrm{BEV}$;

(3) there exists $\mathrm{W}$ such that $\mathrm{EV}=\mathrm{BW}$;

(4) there exists $\mathrm{W}$ such that $\mathrm{EV}=\mathrm{EBW}$;

(5) there exists $\mathrm{W}$ such that $(\mathrm{EBW})_{\mathrm{E}} \subseteq \mathrm{V} \subseteq \mathrm{EBW}$;

(6) the interval $\left[\mathrm{V}_{\mathrm{E}}, \mathrm{EV}\right]$ has a fixed point for the operator $\mathrm{B}$;

(7) $\mathrm{B}\left(\mathrm{V}_{\mathrm{E}}\right) \subseteq \mathrm{EV}$; 
(8) $\mathrm{BV} \subseteq \mathrm{EV}$.

Proof. $(1) \Leftrightarrow(2)$ : Since EV $\subseteq$ BEV $\subseteq$ EBEV, if EV = EBEV, then EV $=$ BEV and we have the direct implication. The converse follows immediately by applying the idempotent operator $\mathrm{E}$ to equality (2).

$(2) \Leftrightarrow(3)$ : The direct implication is trivial. Conversely, if EV = BW for some W, then applying the idempotent operator $\mathrm{B}$, we obtain $\mathrm{BEV}=\mathrm{BW}=\mathrm{EV}$.

$(4) \Leftrightarrow(5)$ : Given W such that EV = EBW, it follows, by Lemma 2.1, that $(\mathrm{EBW})_{\mathrm{E}}=(\mathrm{EV})_{\mathrm{E}}=\mathrm{V}_{\mathrm{E}} \subseteq \mathrm{V} \subseteq \mathrm{EV}=\mathrm{EBW}$. Conversely, if $(\mathrm{EBW})_{\mathrm{E}} \subseteq \mathrm{V} \subseteq \mathrm{EBW}$ for some $W$, then applying the increasing operator $E$, we obtain, by the same lemma, $\mathrm{E}\left((\mathrm{EBW})_{\mathrm{E}}\right)=\mathrm{EBW} \subseteq \mathrm{EV} \subseteq \mathrm{EEBW}=\mathrm{EBW}$, i.e., $\mathrm{EV}=\mathrm{EBW}$.

$(2) \Rightarrow(6) \Rightarrow(4) \Rightarrow(1)$ : The first implication is trivial. For the second one, applying the increasing operator $\mathrm{E}$ to $\mathrm{V}_{\mathrm{E}} \subseteq \mathrm{W}=\mathrm{BW} \subseteq \mathrm{EV}$, we obtain, by Lemma 2.1, the equality $\mathrm{EV}=\mathrm{EBW}$. Finally, if the equality $\mathrm{EV}=\mathrm{EBW}$ holds for some $\mathrm{W}$, then $\mathrm{EBEV}=\mathrm{EBEBW}=\mathrm{EBW}=\mathrm{EV}$, by Corollary 2.6 .

$(6) \Leftrightarrow(7)$ : Let $W$ be a fixed point of the operator $B$ in $\left[V_{E}, E V\right]$. It follows immediately that $\mathrm{B}\left(\mathrm{V}_{\mathrm{E}}\right) \subseteq \mathrm{BW}=\mathrm{W} \subseteq \mathrm{EV}$. Conversely, assuming (7) we have $\mathrm{V}_{\mathrm{E}} \subseteq \mathrm{B}\left(\mathrm{V}_{\mathrm{E}}\right) \subseteq \mathrm{EV}$ and $\mathrm{B}\left(\mathrm{V}_{\mathrm{E}}\right)$ is a fixed point for the operator $\mathrm{B}$.

$(2) \Rightarrow(8) \Rightarrow(7)$ : For the first implication, assuming (2) and applying the operator $\mathrm{B}$ to the inclusion $\mathrm{V} \subseteq \mathrm{EV}$, we obtain $\mathrm{BV} \subseteq \mathrm{BEV}=\mathrm{EV}$. The second implication follows immediately from $\mathrm{B}\left(\mathrm{V}_{\mathrm{E}}\right) \subseteq \mathrm{BV} \subseteq \mathrm{EV}$.

Theorem 3.10. Let $\mathrm{V}$ be a pseudovariety satisfying the conditions of Proposition 3.9. Then $\mathrm{V}$ is E-local.

Proof. It follows immediately from Proposition 3.5, Corollary 3.3 and item (2) from Proposition 3.9.

It is natural to ask whether the conditions of Proposition 3.9 are also necessary. The answer is affirmative when the pseudovariety is contained in EDS.

Theorem 3.11. If $\mathrm{V} \subseteq \mathrm{EDS}$ is an $\mathrm{E}$-local pseudovariety, then items (1)-(8) from Proposition 3.9 hold.

Proof. Let $\mathrm{V} \subseteq$ EDS be an E-local pseudovariety. We prove that $\mathrm{BEV} \subseteq \mathrm{EV}$, as the converse inclusion is trivial. Let $S \in \mathrm{BEV}$, i.e., for every block $B$ of $S,\langle E(B)\rangle \in \mathrm{V}$. Using the E-locality of $\mathrm{V}$, to prove that $S \in \mathrm{EV}$, i.e., $\langle E(S)\rangle \in \mathrm{V}$, it suffices to show that, for every regular $\mathcal{D}$-class $D$ of $S,\langle E(D)\rangle \in \mathrm{V}$. Let $D$ be a regular $\mathcal{D}$-class of $S$. Using again the E-locality of $\mathrm{V}$ and Lemma 2.3, we prove that, for every regular $\mathcal{D}$-class $D^{\prime}$ of $\langle E(D)\rangle,\left\langle E\left(D^{\prime}\right)\right\rangle \in \mathrm{V}$. Recall that, by item (3) from Corollary $2.5, D^{\prime}$ has a unique block, $B^{\prime}$, and is itself a semigroup, since $\mathrm{V} \subseteq$ EDS. Hence $D^{\prime}=B^{\prime}$, for some block $B^{\prime}$ of $\langle E(D)\rangle$. Moreover, there exists a block $B$ of $S$ such that $B^{\prime} \leq B$. Thus $\left\langle E\left(D^{\prime}\right)\right\rangle=\left\langle E\left(B^{\prime}\right)\right\rangle \leq\langle E(B)\rangle \in \mathrm{V}$ (in fact, the equality holds). Hence $\langle E(S)\rangle \in \mathrm{V}$ and $S \in \mathrm{EV}$.

We note that, in the previous theorem, the hypothesis $\mathrm{V} \subseteq$ EDS is mandatory as shown in Example 4.5.

Corollary 3.12. Let $\mathrm{V} \subseteq \mathrm{EDS}$ be such that $\mathrm{V}=\mathrm{EV}$. Then $\mathrm{V}$ is $\mathrm{E}$-local if and only if there exists $\mathrm{W} \subseteq \mathrm{CS}$ such that $\mathrm{V}=\mathrm{BW}$.

Proof. Suppose that V is E-local. Since V = EV and by Theorem 3.11 and item (2) from Proposition 3.9, we have $\mathrm{V}=\mathrm{EV}=\mathrm{BEV}=\mathrm{BV}$. Given $S \in \mathrm{V}=\mathrm{BV}$, we have 
that every block $B$ of $S$ is such that $B \in \mathrm{V} \subseteq \mathrm{EDS}$ and, therefore, $\langle E(B)\rangle \in \mathrm{DS}$, i.e., $\langle E(B)\rangle \in \mathrm{CS}$. Note that $\langle E(B)\rangle$ has the same structure in $\mathcal{R}$-classes and $\mathcal{L}$-classes as $B$, but it may have less elements in the $\mathcal{H}$-classes. Hence $B \in \mathrm{CS}$. It follows that $B \in \mathrm{V} \cap \mathrm{CS}$ and $S \in \mathrm{B}(\mathrm{V} \cap \mathrm{CS})$. The converse follows from B_ being an increasing operator and from $\mathrm{V}=\mathrm{BV}$. Thus, we have $\mathrm{V}=\mathrm{BV}=\mathrm{B}(\mathrm{V} \cap \mathrm{CS})$. The converse implication follows directly from Proposition 3.5.

We suggest, as an easy exercise, the verification of the E-locality of the pseudovarieties J, R, DS and A, for example, using the sufficient conditions of Proposition 3.9.

3.3. The operator _E. Because there are pseudovarieties $\mathrm{V}$ which are not E-local, it is natural to consider the smallest E-local pseudovariety containing $\mathrm{V}$, which we denote $\mathrm{VE}$.

In this subsection, we determine some pseudovarieties of the form $V^{E}$. For that purpose, we also use the operator ${ }_{-E}$ which is studied in detail in [2].

Proposition 3.13. Let $\mathrm{V} \subseteq \mathrm{CS}$ be such that $\mathrm{V}_{\mathrm{E}}=\mathrm{V}$. Then $\mathrm{V}^{\mathrm{E}}=(\mathrm{DV})_{\mathrm{E}}$.

Proof. Let $S=\langle E(S)\rangle \in \mathrm{DV}$ and let W be any E-local pseudovariety containing V. Then $\langle E(D)\rangle \in \mathrm{V}$, for every regular $\mathcal{D}$-class $D$ of $S$, since $D$ is in $\mathrm{V}$ and $\langle E(D)\rangle \leq D$. Since W is E-local and $\mathrm{V} \subseteq \mathrm{W}$, it follows that $S=\langle E(S)\rangle \in \mathrm{W}$. Hence (DV) $\subseteq \subseteq \mathrm{W}$ and, therefore, $(\mathrm{DV})_{\mathrm{E}} \subseteq \mathrm{V}^{\mathrm{E}}$.

For the direct inclusion, since $\mathrm{V} \subseteq \mathrm{CS}$, we have $\mathrm{V} \subseteq \mathrm{DV}$. As $-\mathrm{E}$ is an increasing operator, it follows that $\mathrm{V}=\mathrm{V}_{\mathrm{E}} \subseteq(\mathrm{DV})_{\mathrm{E}}$. By Proposition 3.5, DV is E-local and so is $(\mathrm{DV})_{\mathrm{E}}$, by Corollary 3.3. This yields the inclusion $\mathrm{V}^{\mathrm{E}} \subseteq(\mathrm{DV})_{\mathrm{E}}$.

Corollary 3.14. The class $\mathrm{J}$ is the smallest $\mathrm{E}$-local pseudovariety.

Proof. Let $\mathrm{V}$ be an E-local pseudovariety. Since $\mathrm{I} \subseteq \mathrm{V}$, we have $\mathrm{I}^{\mathrm{E}} \subseteq \mathrm{V}^{\mathrm{E}}=\mathrm{V}$. By Proposition 3.13, we have $\mathrm{I}^{\mathrm{E}}=(\mathrm{DI})_{\mathrm{E}}=J_{\mathrm{E}}=\mathrm{J}$, where the last equality follows from [2, Corollary 5.6]. Hence J $\subseteq$ V. By Example 3.6, J is E-local, which establishes the corollary.

Example 3.15. It follows from Corollary 3.14 that the pseudovarieties LG and CR are not E-local.

Example 3.16. By Proposition 3.13, we conclude that $(R B)^{\mathrm{E}}=(\mathrm{DRB})_{\mathrm{E}}=(\mathrm{DA})_{\mathrm{E}}=$ $\mathrm{DA}$, where the last equality follows by $\left[2\right.$, Corollary 5.6], and $(\mathrm{CS})^{\mathrm{E}}=(\mathrm{DCS})_{\mathrm{E}}=$ $(\mathrm{DS})_{\mathrm{E}}$.

Example 3.17. By Example 3.16, we have $(\mathrm{DS})_{\mathrm{E}}=(\mathrm{CS})^{\mathrm{E}} \subseteq(\mathrm{CR})^{\mathrm{E}}$. Conversely, since $\mathrm{CR} \subseteq \mathrm{DS}$, by [2, Proposition 3.16] and Lemma 2.1, we deduce that $\mathrm{CR}=$ $(\mathrm{CR})_{\mathrm{E}} \subseteq(\mathrm{DS})_{\mathrm{E}}$. Note that $(\mathrm{DS})_{\mathrm{E}}$ is E-local, by Proposition 3.5 and Corollary 3.3. Hence $(C R)^{\mathrm{E}} \subseteq(\mathrm{DS})_{\mathrm{E}}$, which establishes the equality $(\mathrm{CR})^{\mathrm{E}}=(\mathrm{DS})_{\mathrm{E}}$.

Example 3.18. Let $\mathrm{H}$ be a pseudovariety of groups. By [2, Example 3.7], we have $(\mathrm{DH})_{\mathrm{E}}=\mathrm{J} \subseteq \mathrm{J} \vee \mathrm{H} \subseteq \mathrm{DH}$. Since, by Proposition 3.5, DH is E-local, it follows, by Proposition 3.4, that $\mathrm{J} \vee \mathrm{H}$ is E-local. That $\mathrm{J} \vee \mathrm{H}$ is the smallest E-local pseudovariety containing $\mathrm{H}$, is an immediate consequence from the fact that $\mathrm{J}$ is the smallest E-local pseudovariety. Thus $\mathrm{H}^{\mathrm{E}}=\mathrm{J} \vee \mathrm{H}$.

Example 3.19. Let $\mathrm{H}$ be a pseudovariety of groups. By Example 3.16, we have $\mathrm{DA}=(\mathrm{RB})^{\mathrm{E}} \subseteq(\mathrm{RB} \vee \mathrm{H})^{\mathrm{E}}$ and, therefore, $\mathrm{DA} \vee \mathrm{H} \subseteq(\mathrm{RB} \vee \mathrm{H})^{\mathrm{E}}$. Now, from [2, Lemma 3.1, Example 3.8, Corollary 4.2], we obtain $(\mathrm{DO} \cap \overline{\mathrm{H}})_{\mathrm{E}} \subseteq(\mathrm{DO})_{\mathrm{E}} \cap \overline{\mathrm{H}}_{\mathrm{E}}=$ 
$\mathrm{DA} \cap \overline{\mathrm{H}} \subseteq \mathrm{DA}$. Therefore, $(\mathrm{DO} \cap \overline{\mathrm{H}})_{\mathrm{E}} \subseteq \mathrm{DA} \subseteq \mathrm{DA} \vee \mathrm{H} \subseteq \mathrm{DO} \cap \overline{\mathrm{H}}$. As an intersection of E-local pseudovarieties is E-local, the pseudovariety $D O \cap \overline{\mathrm{H}}$ is E-local. By Proposition 3.4, DA $\vee \mathrm{H}$ is E-local. Thus $(\mathrm{RB} \vee \mathrm{H})^{\mathrm{E}}=\mathrm{DA} \vee \mathrm{H}$.

Example 3.20. Since LG $\subseteq$ DS and DS is E-local by Proposition 3.5, we have $(\mathrm{LG})^{\mathrm{E}} \subseteq \mathrm{DS}$. On the other hand, by Example 3.16 and by [2, Example 3.17], we obtain $(\mathrm{DS})_{\mathrm{E}}=(\mathrm{CS})^{\mathrm{E}}=\left((\mathrm{LG})_{\mathrm{E}}\right)^{\mathrm{E}} \subseteq(\mathrm{LG})^{\mathrm{E}}$. Thus the equality $(\mathrm{DS})_{\mathrm{E}} \subseteq(\mathrm{LG})^{\mathrm{E}} \subseteq \mathrm{DS}$ holds.

If we prove that $(D S)_{E}=D S$, we will have the equality in the previous example. This provides additional motivation for the calculation of $(D S)_{E}$ which remains an open problem (see [2]).

We end this subsection by noting that $(\mathrm{V} \cap \mathrm{W})^{\mathrm{E}} \subseteq \mathrm{V}^{\mathrm{E}} \cap \mathrm{W}^{\mathrm{E}}$, for all pseudovarieties $\checkmark$ and $W$. However, we do not know whether equality holds.

\section{E-LOCAL PSEUdoidentities}

We call E-local a pseudoidentity which defines an E-local pseudovariety. Note that a pseudovariety defined by a set of E-local pseudoidentities is E-local, since it is the intersection of the E-local pseudovarieties defined by each pseudoidentity of the set. We do not know whether the converse is valid.

Our results from the previous section yield several E-local pseudoidentities. However, some of the results that we obtained, like some techniques developed allow us to give a different characterization of several types of pseudoidentities with this property.

For $u \in \bar{\Omega}_{A} \mathrm{~S}$, let first $(u)$ and last $(u)$ be, respectively, the first and last letters of $u$. We relate the E-locality of the pseudoidentities of the form $u=v$, where first $(u) \neq \operatorname{first}(v)$ or last $(u) \neq \operatorname{last}(v)$, with the condition $\mathrm{V} \subseteq \llbracket u=v \rrbracket$, where $\mathrm{V}$ is one of the pseudovarieties $\mathrm{R}, \mathrm{L}$ and $\mathrm{J}$. We also obtain some results concerning the pseudovariety DA.

Proposition 4.1. The following properties are verified by every pseudoidentity $u=v$.

(1) If last $(u) \neq \operatorname{last}(v)$ and $\mathrm{R}$ satisfies $u=v$, then $u=v$ is $\mathrm{E}$-local.

(2) If first $(u) \neq \operatorname{first}(v)$ and $\mathrm{L}$ satisfies $u=v$, then $u=v$ is $\mathrm{E}$-local.

(3) If $\operatorname{first}(u) \neq \operatorname{first}(v)$, last $(u) \neq \operatorname{last}(v)$ and $\mathrm{J}$ satisfies $u=v$, then $u=v$ is E-local.

Proof. Let $u=v$ be a pseudoidentity such that last $(u) \neq \operatorname{last}(v)$ and suppose that $\mathrm{R}$ satisfies $u=v$. We claim that $\mathrm{R} \subseteq \llbracket u=v \rrbracket \subseteq$ ER. So that, by Example 3.7 and by Proposition 3.4, $\llbracket u=v \rrbracket$ is E-local. The first inclusion is assumed by hypothesis. To prove the second inclusion, let $S$ be a semigroup satisfying $u=v$ and suppose that $S \notin$ ER, i.e., $\langle E(S)\rangle \notin$ R. Then, by [1, cf. Exercise 5.2.8], there exist two distinct idempotents such that $e f=f$ and $f e=e$. Evaluating the last letter of $u$ by $e$, the last letter of $v$ by $f$ and the other letters by $e$ or $f$, we obtain that $S$ satisfies $e=u=v=f$, which is a contradiction.

Similarly, we obtain (2) and (3).

Since, by [2], the pseudovarieties R, L, J and DA satisfy the equality $V_{E}=V$, it is easy to obtain the following results:

Theorem 4.2. Let $u=v$ be an arbitrary pseudoidentity. 
(1) If first $(u)=\operatorname{first}(v)$ and $\operatorname{last}(u) \neq \operatorname{last}(v)$, then $u=v$ is $\mathrm{E}$-local if and only if $\mathrm{R}$ satisfies $u=v$.

(2) If $\operatorname{first}(u) \neq \operatorname{first}(v)$ and last $(u)=\operatorname{last}(v)$, then $u=v$ is $\mathrm{E}$-local if and only if $\mathrm{L}$ satisfies $u=v$.

(3) If $\operatorname{first}(u) \neq \operatorname{first}(v)$ and last $(u) \neq \operatorname{last}(v)$, then $u=v$ is $\mathrm{E}$-local if and only if $\mathrm{J}$ satisfies $u=v$.

(4) If first $(u)=\operatorname{first}(v)$, last $(u)=\operatorname{last}(v)$ and $u=v$ is $\mathrm{E}$-local, then DA satisfies $u=v$.

Proof. (1) Let $u=v$ be a pseudovariety $\operatorname{such}$ that $\operatorname{first}(u)=\operatorname{first}(v)$ and $\operatorname{last}(u) \neq$ last $(v)$. Suppose that it is E-local. Since $\mathrm{LZ} \subseteq \mathrm{CS}$ and $(\mathrm{LZ})_{\mathrm{E}}=\mathrm{LZ}$, it follows from Proposition 3.13 that $(L Z)^{\mathrm{E}}=(\mathrm{DLZ})_{\mathrm{E}}=\mathrm{R}_{\mathrm{E}}=\mathrm{R}$. Thus, as the pseudovariety $\llbracket u=v \rrbracket$ is E-local and it contains $\mathrm{LZ}$, it also contains $(\mathrm{LZ})^{\mathrm{E}}=\mathrm{R}$. The converse follows from Proposition 4.1. Dually, we obtain (2).

(3) It follows directly from $\mathrm{J}$ being the smallest E-local pseudovariety (see Corollary 3.14) and from Proposition 4.1.

(4) In that case, we have $\mathrm{RB} \models u=v, \mathrm{RB} \subseteq \mathrm{CS}$ and $(\mathrm{RB})_{\mathrm{E}}=\mathrm{RB}$. By Proposition 3.13, we have $(R B)^{E}=(D R B)_{E}=(D A)_{E}=D A$. As in (1), we deduce that $\mathrm{DA}=(\mathrm{RB})^{\mathrm{E}} \subseteq \llbracket u=v \rrbracket$.

However, we do not have a characterization of all E-local pseudoidentities of the form $u=v$, $\operatorname{with} \operatorname{first}(u)=\operatorname{first}(v)$ and last $(u)=\operatorname{last}(v)$.

Theorem 4.3 provides another sufficient condition for a pseudoidentity to be E-local that follows from Lemma 2.4.

Theorem 4.3. Let $u=v$ be a pseudoidentity such that $u, v \in \overline{\langle X\rangle}$, where all elements of $X \subseteq \bar{\Omega}_{A} \mathrm{~S}$ lie in a same regular $\mathcal{D}$-class of $\bar{\Omega}_{A} \mathrm{~S}$. Then $u=v$ is $\mathrm{E}$-local.

Proof. Let $S$ be a finite semigroup and suppose that $\langle E(D)\rangle \models u=v$, for each regular $\mathcal{D}$-class $D$ of $S$. We want to prove that $\langle E(S)\rangle \models u=v$. Let $\varphi: \bar{\Omega}_{A} S \rightarrow S$ be a continuous surjective homomorphism such that, for every $x \in X, \varphi(x) \in\langle E(S)\rangle$. Since all elements of $X$ lie in a same regular $\mathcal{D}$-class of $\bar{\Omega}_{A} \mathrm{~S}$, then there exists a regular $\mathcal{D}$-class $D$ of $S$ such that $\varphi(x) \in D$, for all $x \in X$. By Lemma 2.4, it follows that $\varphi(x) \in\langle E(D)\rangle$, for all $x \in X$. Since $u, v \in \overline{\langle X\rangle}$, it follows that $\varphi(u), \varphi(v) \in\langle E(D)\rangle$ and, by hypothesis, they are equal. Thus $\langle E(S)\rangle \models u=v$ and $u=v$ is E-local.

Note that several pseudoidentities considered in this paper are of this form. Specifically, the pseudoidentities that we used in Section 2 to define the pseudovarieties J, R, L, A, DA, DG, DO and DS are all of this form. Another example is the pseudoidentity $\left(x^{\omega} y^{\omega}\right)^{\omega}=\left(y^{\omega} x^{\omega}\right)^{\omega}$ which defines the pseudovariety BG. As a last example, Almeida and Volkov [3] showed that, if $u_{i}=v_{i}$, with $i \in I$, is a basis of pseudoidentities for a pseudovariety of groups $\mathrm{H}$, then $u_{i}^{\prime}=v_{i}^{\prime}$ is a basis of pseudoidentities for $\overline{\mathrm{H}}$, where $u_{i}^{\prime}$ and $v_{i}^{\prime}$ result from the substitution of each letter $x_{j} \in A$ of $u_{i}$ and $v_{i}$ by $e x_{j} e$ where $e$ is a fixed idempotent in the minimum ideal of $\bar{\Omega}_{A}$ S. These pseudoidentities are also of the form of Theorem 4.3.

In the last result, we identify all E-local pseudoidentities with only one variable. The elements of these equalities are elements of the free profinite semigroup on the alphabet with a unique element $x$, and so they can be written under the form $x^{\alpha}$, where $\alpha$ is an element of the profinite completion of $(\mathbb{N},+)$. We use the term infinite to refer to the elements of this semigroup which are not in $\mathbb{N}$. 
Corollary 4.4. The E-local pseudoidentities in one variable are those of the form $x^{\alpha}=x^{\beta}$, with both $\alpha$ and $\beta$ infinite.

Proof. If $\alpha$ or $\beta$ are finite and are not equal, then DA does not satisfy the pseudoidentity $u=v$ since DA contains all finite monogenic aperiodic semigroups. Thus, $u=v$ is not E-local, by item (4) of Theorem 4.2.

On the other hand, if $\alpha$ and $\beta$ are infinite, then $x^{\alpha}$ and $x^{\beta}$ are in a same group with neutral element $x^{\omega}$. Thus, by Theorem 4.3, the pseudoidentity is E-local.

We do not know if every E-local pseudovariety is defined by a set of pseudoidentities satisfying the condition of Theorem 4.3.

We finish the paper going back to Section 3.2, where we asked whether Theorem 3.11 can be generalized to all E-local pseudovarieties. The answer is negative as we see in the following example.

Example 4.5. Consider the pseudovariety $W=\llbracket\left((x y)^{\omega} x(x y)^{\omega}\right)^{2}=(x y)^{\omega} x(x y)^{\omega} \rrbracket$, which is E-local by Theorem 4.3. We show that $W$ does not satisfy the condition $\mathrm{BV} \subseteq \mathrm{EV}$ from Proposition 3.9.

Let $S$ be the syntactic semigroup of the regular language $\left(a^{+} b^{+} c^{+} d^{+}\right)^{+} a^{+} b^{+}$. $\left(a^{+} b^{+} c^{+} d^{+}\right)^{+}$, whose egg-box picture is presented in Figure 1. The blocks of $S$ are the trivial semigroup and the unique block of the unique non-trivial regular $\mathcal{D}$-class of $S, D$, and which we denote by $B$. It is easy to see that $B \in \mathrm{W}$. Note that $(x y)^{\omega} x$ and $(x y)^{\omega}$ define implicitly $\mathcal{R}$-equivalent elements, the latter being an idempotent. Now, the product in $B$ of two $\mathcal{R}$-equivalent elements of $D$ with the second one an idempotent is an idempotent in $D$ or is 0 . On the other hand, the elements $a b c d a b$ and $a b c d$ are $\mathcal{R}$-equivalent and the second one is an idempotent, but their product in $S, a b c d a b a b c d$, is not an idempotent (it belongs to the 0-minimal and non-regular $\mathcal{D}$-class of $S$ ). Thus $S=\langle E(S))\rangle \notin \mathrm{W}$, i.e., $S \notin \mathrm{EW}$.

Acknowledgments. The author is grateful to the anonymous referee for warning a flaw in the proof of the initially statement of Theorem 3.11. This work is part of the author's doctoral thesis, written under the supervision of Prof. Jorge Almeida, from whose advice the author has greatly benefited. This work was supported by Fundação para a Ciência e a Tecnologia (FCT) through the PhD Grant $\mathrm{SFRH} / \mathrm{BD} / 19720 / 2004$, through the Centro de Matemática da Universidade do Porto (CMUP) and also through the project PTDC/MAT/65481/2006, which is partly funded by the European Community Fund FEDER.

\section{REFERENCES}

[1] J. Almeida, Finite Semigroups and Universal Algebra, World Scientific, Singapore, 1994. English translation.

[2] J. Almeida and A. Moura, Idempotent-generated semigroups and pseudovarieties, Proc. Edinburgh Math. Soc. 54 (2011) 545-568.

[3] J. Almeida and M. V. Volkov, Profinite identities for finite semigroups whose subgroups belong to a given pseudovariety, J. Algebra and Applications 2 (2003) 137-163.

[4] D. G. Fitz-Gerald, On inverses of products of idempotents in regular semigrups, J. Aust. Math. Soc. 13 (1972) 335-337.

[5] R. Graham, On finite 0-simple semigroups and graph theory, Math. Syst. Theory 2 (1968) 325-339.

[6] J.-E. Pin, Varieties of Formal Languages, Plenum, London, 1986. English translation.

[7] $\longrightarrow$, PG = BG, a success story, in NATO Advanced Study Institute Semigroups, Formal Languages and Groups, J. Fountain, ed., Kluwer academic publishers, 1995, 33-47.

[8] J. Reiterman, The Birkhoff theorem for finite algebras, Algebra Universalis 14 (1982) 1-10. 


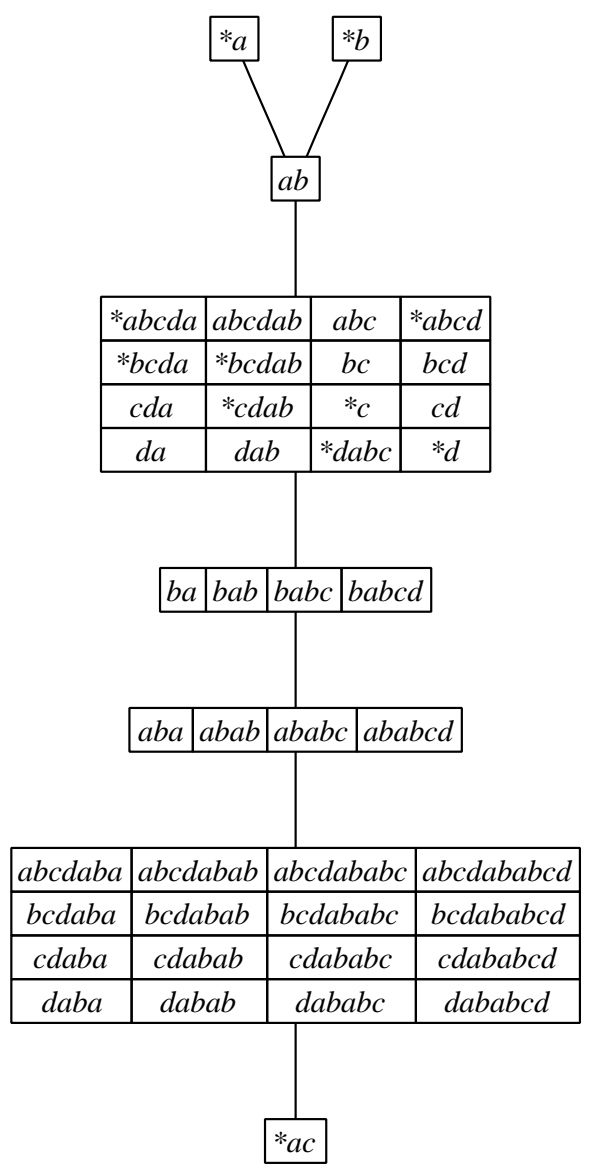

FIGURE 1. The egg-box picture of a semigroup in BW and not in EW

[9] J. Rhodes and B. Steinberg, The q-theory of finite semigroups, Springer, New York, 2009.

[10] B. Tilson, Complexity of two J-class semigroups, Advances Math. 11 (1973) 215-237.

Instituto Superior de Engenharia do Porto/LEMA and Centro de Matemática da Universidade do Porto, Rua do Campo Alegre, 687, 4169-007 Porto, Portugal

E-mail address: aim@isep.ipp.pt/amoura@fc.up.pt 